\title{
Catching a glimpse of the parton structure of the bound proton
}

\author{
Sara Fucini, ${ }^{1}$ Sergio Scopetta, ${ }^{1}$ and Michele Viviani ${ }^{2}$ \\ ${ }^{1}$ Dipartimento di Fisica e Geologia, Università degli Studi di Perugia and Istituto Nazionale di Fisica Nucleare, \\ Sezione di Perugia, via A. Pascoli, I - 06123 Perugia, Italy \\ 2 INFN-Pisa, 56127 Pisa, Italy
}

(Dated: March 26, 2020)

\begin{abstract}
A new generation of experiments is expected to shed light on the elusive parton structure of the bound proton. One of the most promising directions is incoherent deeply virtual Compton scattering, which can provide a tomographic view of the bound proton. The first measurement has been recently performed, using ${ }^{4} \mathrm{He}$ targets at Jefferson Lab. In the work presented here, a rigorous Impulse Approximation analysis of this process is proposed. As ingredients, state-of-the-art models of the nuclear spectral function and of the parton structure of the struck proton, together with novel scattering amplitudes expressions for a bound moving nucleon, have been used. The overall agreement obtained with the data, good in particular at high values of the photon virtuality, demonstrates the solidity of the framework, which is also suitable for further improvements. It is found that possible big differences between results for the bound proton and those for the free one could be related to kinematical nuclear effects and not to modifications of the parton structure. The analysis demonstrates that the comparison of the results of this approach, based on a conventional description, with future precise data, has the potential to expose exotic quark and gluon effects in nuclei.

PACS numbers: 13.60.Hb,14.20.Dh,27.10.+h

Keywords: Deeply Virtual Compton scattering, bound proton parton structure
\end{abstract}

Several decades ago, the discovery of the European Muon collaboration (EMC) effect in inclusive deep inelastic scattering off nuclear targets [1] has shown that the parton structure of bound nucleons is modified by the nuclear medium (see Ref. 2] for a recent report). A new generation of planned measurements at high energy and high luminosity facilities could provide in the next years, for the first time, a fully quantitative explanation of the EMC effect (for a recent report, see, e.g., Ref. [3, 4). This programme includes the challenging realization of semi-inclusive and exclusive experiments and their complicated theoretical description. Among the most promising directions, nuclear deeply virtual Compton scattering (DVCS), the hard exclusive leptoproduction of a real photon on a nuclear target, plays a special role. In DVCS, the parton structure is encoded in the so called generalized parton distributions (GPDs) [5, non perturbative quantities providing a wealth of novel information (for exhaustive reports see, e.g., Ref. [6]). Nuclear DVCS could unveil the presence of non-nucleonic degrees of freedom 7, or may allow to better understand the distribution of nuclear forces in nuclei [8]. Nonetheless, the subject of this letter is mostly related to the tomography of the bound proton, i.e., the distribution of partons with a given longitudinal momentum in the transverse plane. This is certainly one of the most exciting information accessible in DVCS through the GPDs formalism [9]. In nuclei, DVCS can occur through two different mechanisms, i.e., the coherent one $A\left(e, e^{\prime} \gamma\right) A$, where the target $A$ remains intact recoiling as a whole, and the incoherent one $A\left(e, e^{\prime} \gamma p\right) X$, where the nucleus breaks up and the struck proton is detected, so that its tomography could be ultimately obtained. The comparison between this information and that obtained for the free proton could provide a pictorial view of the realization of the EMC effect. From an experimental point of view, the study of nuclear DVCS requires the very difficult coincidence detection of fast photons and electrons together with slow, intact recoiling protons or nuclei. For this reason, in the first measurement of nuclear DVCS at HERMES 10, a clear separation was not achieved between the two different DVCS channels. Nevertheless, recently, for the first time, such a separation has been performed by the EG6 experiment of the CLAS collaboration [11, with the $6 \mathrm{GeV}$ electron beam at Jefferson Lab (JLab). The first data for coherent and incoherent DVCS off ${ }^{4} \mathrm{He}$ have been published in Refs. [12] and 13], respectively. Among few nucleon systems, for which a realistic evaluation of conventional nuclear effects is possible in principle, ${ }^{4} \mathrm{He}$ is deeply bound and represents the prototype of a typical finite nucleus. Realistic approaches allow to distinguish conventional nuclear effects from exotic ones, which could be responsible of the observed EMC behaviour. Without realistic benchmark calculations, the interpretation of the data will be hardly conclusive. In fact, in Refs. 12, 13, the importance of new calculations has been addressed, for a successful interpretation of the collected data and of those planned at JLab in the next years [14. In facts available estimates, proposed long time ago, correspond in some cases to different kinematical regions [15, 16]. We have therefore recently performed a successful impulse approximation (IA) analysis of coherent DVCS off 
${ }^{4} \mathrm{He}$ [17, obtaining an overall good agreement with the data [12. In this letter, we propose an analogous analysis for the incoherent channel, to see to what extent a conventional description can describe the recent data 13 which have the tomography of the bound proton as the ultimate goal. One should notice that, in Ref.[17, the calculation of the coherent channel required, as the only theoretical tool, the nuclear GPD. In the present investigation completely new issues arise, such as the calculation of the appropriate differential cross sections for a moving proton in the medium, and the modelling of a diagonal nuclear spectral function.

We studied therefore the IA to the handbag approximation to the incoherent DVCS process, $A\left(e, e^{\prime} \gamma p^{\prime}\right) X$, shown in Fig. 1. It means that we assumed that the process goes through one quark in one nucleon in ${ }^{4} \mathrm{He}$, i.e., non-nucleonic degrees of freedom are not considered, and further possible rescattering of the struck detected proton with the remnant $X$ is disregarded. For high enough values of the initial photon virtuality, $Q^{2}=$ $-q_{1}^{2}=-\left(k-k^{\prime}\right)^{2}$, IA usually provides the bulk of nuclear effects in a hard electron scattering process (see, e.g., Ref. 18 for an experimental study of the onset of the validity of IA calculations). Similar expectations hold in the present study, although only the comparison with data can demonstrate the validity of the chosen framework. If $Q^{2}$ is much larger than $-t=-\Delta^{2}=-\left(p-p^{\prime}\right)^{2}$, the momentum transferred to the hadronic system with initial (final) 4-momentum $p\left(p^{\prime}\right)$, the hard vertex of the "handbag" diagram depicted in Fig. 1 can be studied perturbatively. The soft part is parametrized in terms of GPDs of the struck proton, which depend on $\Delta^{2}$, on the so-called skewness $\xi=-\Delta^{+} / P^{+}$, i.e., the difference in plus momentum fraction between the initial and the final states, and on $x$, the average plus momentum fraction of the struck parton with respect to the total momentum, not experimentally accessible (the notation $a^{ \pm}=\left(a_{0} \pm a_{3}\right) / \sqrt{2}$ is used). The average four momenta, for photons and protons, are $q=\left(q_{1}+q_{2}\right) / 2$ and $P=p+p^{\prime}$, respectively. In IA, one also has $-\Delta^{2}=-\left(q_{1}-q_{2}\right)^{2}$, that is, the momentum transferred to the system coincides with that transferred to the struck proton. The reference frame proposed in Ref. [19, with the target at rest, the virtual photon with energy $\nu$ moving opposite to the $\hat{z}$ axis and the leptonic and hadronic planes of the reaction defining the angle $\phi$, has been adopted. Besides, using energy-momentum conservation, one gets for the azimuthal angle of the detected proton the relation $\phi_{p^{\prime}}=\phi+\phi_{e}$ and, since in the chosen frame $\phi_{e}=0, \phi_{p^{\prime}}$ coincides with $\phi$. A pure DVCS process always interferes with the electromagnetic Bethe-Heitler (BH) process, which produces the same final state $\left(e^{\prime} \gamma p^{\prime}\right)$. The IA description of the BH process is shown in Fig. 2. We note in passing that the possibility that the real photon is emitted by the initial nucleus, or by the final $\mathrm{X}$ system, has been neglected, being the BH cross-section

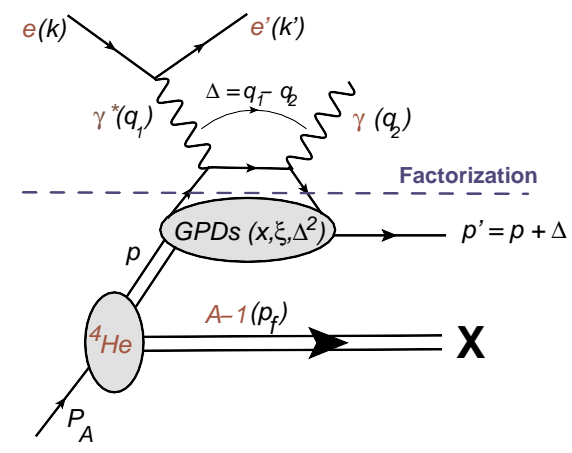

FIG. 1: (color online) Incoherent DVCS process off ${ }^{4} \mathrm{He}$ in the IA to the handbag approximation.

approximately proportional to the inverse squared mass of the emitter. With respect to the emission from the electrons, this contribution should be therefore negligibly small. For this reason, the experimental collaboration EG6 has not considered this occurrence in its analysis. From a theoretical point of view, neglecting these contributions, gauge invariance is not reproduced. Nonetheless we have to point out that in the present Impulse Approximation analysis gauge invariance is in any case not fulfilled and it could be restored at the nuclear level only implementing many-body currents. These corrections have not been included yet in the calculation and they could be more relevant than photon emission from nuclear systems. Since GPDs are not directly measurable, the experimental way to access their physical content exploits the BH-DVCS interference. In facts, in the squared amplitude of the process under scrutiny,

$$
\mathcal{A}^{2}=T_{D V C S}^{2}+T_{B H}^{2}+\mathcal{I}
$$

in the kinematical region of the performed experiment, the $\mathrm{BH}$ mechanism is dominating on the DVCS one. By measuring the beam-spin asymmetry (BSA) of the process off an unpolarized (U) target

$$
A_{L U}=\frac{d \sigma^{+}-d \sigma^{-}}{d \sigma^{+}+d \sigma^{-}}
$$

where \pm refers to positive/negative longitudinal (L) beam helicity, in a leading-twist analysis it is possible to isolate the BH-DVCS interference $\mathcal{I}=2 \Re e\left(T_{D V C S} T_{B H}^{*}\right)$. This term is sensitive to the target partonic content, parametrized through GPDs hidden in the so called Compton Form Factors (CFF), appearing in the $T_{D V C S}$ amplitude. We studied therefore the BSA, the observable recently measured at JLab and a workable expression for it is needed.

Let us describe our IA calculation of the BSA. To evaluate Eq. (2), the cross-section for a DVCS process occurring off a bound moving proton in ${ }^{4} \mathrm{He}$ is required. In our IA approach, the off-shellness of the initial bound proton is purely kinematical, i.e., the energy of the struck 

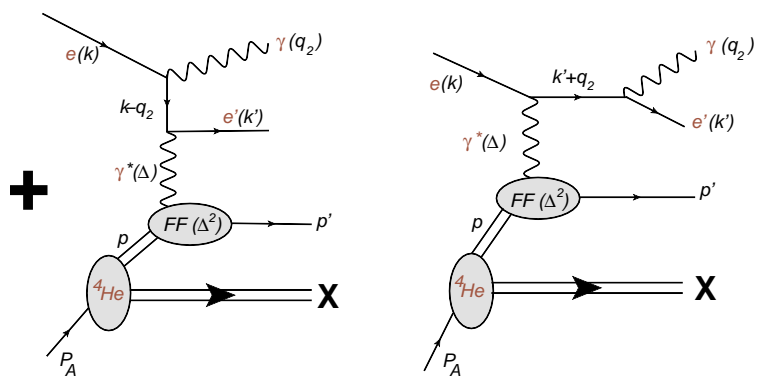

FIG. 2: (color online) The Bethe Heitler process in IA.

proton is obtained from energy conservation and reads

$$
p_{0}=M_{A}-\sqrt{M_{A-1}^{* 2}+\vec{p}^{2}} \simeq M-E-T_{r e c},
$$

where we define the removal energy $E=M_{A-1}^{*}+M-$ $M_{A}=\epsilon_{A-1}^{*}+\left|E_{A}\right|-\left|E_{A-1}\right|$ in terms of the binding energy (mass) of ${ }^{4} \mathrm{He}$ and of the 3 -body system, $E_{A}\left(M_{A}\right)$ and $E_{A-1}\left(M_{A-1}^{*}\right)$, respectively, and of the excitation energy of the recoiling system, $\epsilon_{A-1}^{*}$. Finally, $T_{r e c}$ is the kinetic energy of the recoiling 3 -body system and $M$ is the proton mass. In this way, after a straightforward but lengthy analysis, which will be shown elsewhere 20], one finds a complicated convolution formula for the cross section, which can be sketched as follows

$$
d \sigma_{\text {Inc }}^{ \pm}=\int_{\text {exp }} d E d \vec{p} \frac{p \cdot k}{p_{0}|\vec{k}|} P^{4} H e(\vec{p}, E) d \sigma_{b}^{ \pm}(\vec{p}, E, K),
$$

in terms of the nuclear spectral function $P^{4} \mathrm{He}(\vec{p}, E)$ and of the cross section for a DVCS process off a bound proton, $d \sigma_{b}^{ \pm}$. The integral on the removal energy refers to both discrete and continuous energy spectra of ${ }^{4} \mathrm{He}$. In Eq. (4), $K$ is the set of kinematical variables $\left\{x_{B}=\right.$ $\left.Q^{2} /(2 M \nu), Q^{2}, t, \phi\right\}$. The range of $K$ accessed in the experiment fixes the proper energy and momentum integration space, denoted as exp. From Eq. (4) we get the measured differential cross section, appearing in Eq. (2),

$$
\begin{aligned}
d \sigma^{ \pm} \equiv \frac{d \sigma_{I n c}^{ \pm}}{d x_{B} d Q^{2} d \Delta^{2} d \phi} & =\int_{\exp } d E d \vec{p} P^{4} H e(\vec{p}, E) \\
& \times\left|\mathcal{A}^{ \pm}(\vec{p}, E, K)\right|^{2} g(\vec{p}, E, K),
\end{aligned}
$$

where $g(\vec{p}, E, K)$ is a complicated function which arises from the integration over the phase space and includes also the flux factor $p \cdot k /\left(p_{0}|\vec{k}|\right)$ in Eq. (4). This latter term comes from the fact that one has at disposal only non-relativistic wave functions to evaluate the spectral function. This implies also that either the number or the momentum sum rule is slightly violated. Such a problem could be solved with a Light Front approach, as proposed in Ref. 21] for a 3-body system. The BSA assumes the schematic form

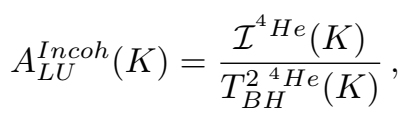

where

$$
\begin{gathered}
\mathcal{I}^{4} H e(K)=\int_{\text {exp }} d E d \vec{p} P^{4} H e(\vec{p}, E) g(\vec{p}, E, K) \mathcal{I}(\vec{p}, E, K), \\
T_{B H}^{2{ }^{4} H e}(K)=\int_{\exp } d E d \vec{p} P^{4} H e(\vec{p}, E) g(\vec{p}, E, K) \\
\times T_{B H}^{2}(\vec{p}, E, K),
\end{gathered}
$$

where $\mathcal{I}$ and $T_{B H}^{2}$ refer to a moving bound nucleon and generalize the Fourier decomposition of the DVCS cross section off a proton at rest, at leading twist, derived in Ref. [19]. The new expressions for $\mathcal{I}$ and $T_{B H}^{2}$ will be presented elsewhere [20]; here, without going into technical details, we summarize the structure of the different contributions. For the $\mathrm{BH}$ part, we considered the full sum of azimuthal harmonics, i.e $T_{B H}^{2}=c_{0}^{\text {bound }}+c_{1}^{\text {bound }} \cos \phi+c_{2}^{\text {bound }} \cos (2 \phi)$, where the coefficients $c_{i}^{\text {bound }}$ contain the Dirac and Pauli form factors (FFs). This decomposition is driven by the explicit form of the $\mathrm{BH}$ propagators shown in Fig. 2. We stress that in the present IA approach no nuclear modifactions occur for the FFs of the bound proton. As for the interference term, we considered the leading twist contribution, so that terms explicitly proportional to $\Delta^{2} / Q^{2}$ 22. have been neglected while corrections proportional to $\epsilon^{2}$, with $\epsilon=2 M x_{B} / Q$, accounting for target mass corrections, have been considered. In the numerator of Eq. 22 only the term accounting for the beam polarization is selected, where the dependence on the parton structure of the bound proton is hidden in the imaginary part of the CFF $\mathcal{H}$. In the kinematics of interest, this quantity can be expressed in terms of only one GPD of the bound proton, $H\left(x, \xi, \Delta^{2}\right)$, according to $\Im m \mathcal{H}\left(\xi^{\prime}, t\right)=H\left(\xi^{\prime}, \xi^{\prime}, t\right)-H\left(-\xi^{\prime}, \xi^{\prime}, t\right)$. We notice that the off-shellness affects the proton parton structure, since the GPDs have to be evaluated for a skewness $\xi^{\prime}=Q^{2} /(2 P \cdot q)$ given in terms of the 4-momenta of the proton and the photons. The modification at partonic level is due to this rescaling of the skewness that, for a proton at rest, reduces to $\xi \simeq x_{B} /\left(2-x_{B}\right)$.

In order to actually evaluate Eq. (6), we need an input for the proton GPD and for the nuclear spectral function. Concerning the nuclear part, only old attempts exist of obtaining a spectral function of ${ }^{4} \mathrm{He}[23,24$. Its realistic evaluation would require the knowledge, at the same time, of exact solutions of the Schrödinger equation with realistic nucleon-nucleon potentials and three-body forces for the ${ }^{4} \mathrm{He}$ nucleus and for the three-body recoiling system, which can be also unbound with an excitation energy $\epsilon_{A-1}^{*}$. This latter part represents a very complicated few-body problem, whose solution is presently unknown. A full realistic calculation of the ${ }^{4} \mathrm{He}$ spectral function is planned and has started but, in this work, for $P^{4} \mathrm{He}(\vec{p}, E)$ use is made of the model presented in Ref. [25, 26]. In particular, when the recoiling system is in 


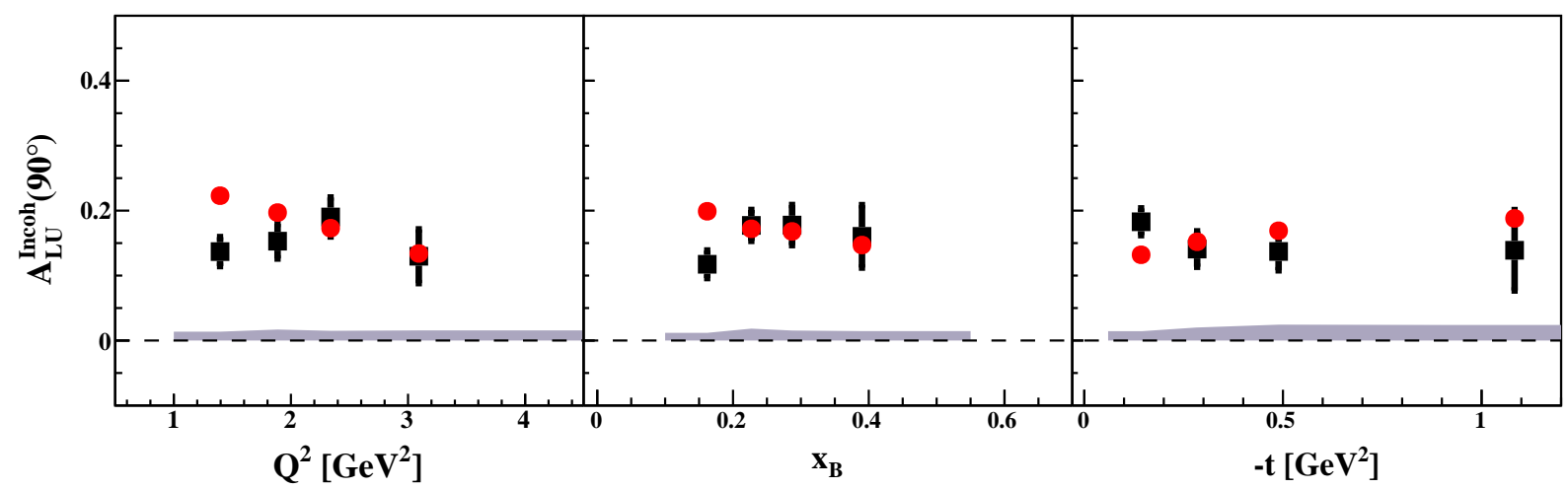

FIG. 3: (Color online) Azimuthal beam-spin asymmetry for the proton in ${ }^{4} \mathrm{He}, A_{L U}^{I n c o h}(K)$, for $\phi=90^{\circ}$ : results of this approach (red dots) compared with data (black squares) [12. From left to right, the quantity is shown in the experimental $Q^{2}, x_{B}$ and $t$ bins, respectively. Shaded areas represent systematic errors.

its ground state, an exact description is used in terms of variational wave functions for the 4-body and 3-body systems, obtained through the hyperspherical harmonics method [27], within the Av18 NN interaction [28], including UIX three-body forces 29. The cumbersome part of the spectral function, with the recoiling system excited, is based on the Av18+UIX interaction, proposed in Ref. [25, 26], an update of the two-nucleon correlation model of Ref. 30. We note in passing that a realistic calculation of GPDs for ${ }^{3} \mathrm{He}$ has been completed, where the importance of the $E$-dependence of the spectral function has been established (see Ref. [31] and references there in). Clearly our final results will depend on the adopted model for the spectral function. We stress anyway that, since other available models are less realistic than the one exploited here, their use would not add interest to the present analysis. As already said, we are actively working to obtain an exact spectral function.

For the nucleonic GPD, the model of Goloskokov and Kroll (GK) 32 has been used, as we did succesfully in the coherent case [17. We remind that the model is valid in principle at $Q^{2}$ values larger than those of interest here, in particular at $Q^{2} \geq 4 \mathrm{GeV}^{2}$. Nonetheless we checked that the GK model can reasonably describe free proton data collected in similar kinematical ranges [33. We therefore adopt it, also because other workable global models of GPDs are not easily available. The study of the dependence of our results on proton GPDs models is beyond the scope of the present letter and will be presented elsewhere [20].

With these ingredients at hand, Eq. 60 can be evaluated and the comparison with the recent data [13] is possible. The BSA is a function of the azimuthal angle $\phi$ and of the kinematical variables $Q^{2}, x_{B}$ and $t$. Due to limited statistics, in the experimental analysis these latter variables have been studied separately with a two- dimensional data binning. The same procedure has been used in our calculation. For example, each point at a given $x_{B}$ has been obtained using for $t$ and $Q^{2}$ the corresponding average experimental values. In Fig. 3 it is seen that, overall, the calculation reproduces the data rather well. In particular, the agreement is not satisfactory only in the region of low $Q^{2}$. Indeed, this is evident only in the experimental points corresponding to the lowest values of $Q^{2}, x_{B}$ and $t$. One should notice that the average value of $Q^{2}$ grows with increasing $x_{B}$ and $t$, so that a not satisfactory description at low $Q^{2}$ affects also the first $x_{B}$ and $t$ bins. A careful analysis of the interplay between the $t$ and $Q^{2}$ dependence of the data is required to establish whether final state interaction (FSI) effects, beyond IA, could be responsible of this disagreement. In the light of this comparison, we can conclude that a careful use of basic conventional ingredients is able to reproduce the data. In order to better understand our results disentangling nuclear modifications possibly related to the EMC effect, as an illustration we divide our BSA by the corresponding free proton quantity, as it is given in the literature (see, e.g., Eq. (2) in Ref. 34]), based on the GK model used in the calculation, and we plot it as a function of $x_{B}$. As it is seen in Fig. 4, an effect as big as 25 percent is found. Is that a medium modification of the parton structure? Actually, such a ratio can be sketched as follows

$$
\frac{A_{L U}^{I n c o h}}{A_{L U}^{p}}=\frac{\mathcal{I}^{4} H e}{\mathcal{I}^{p}} \frac{T_{B H}^{2 p}}{T_{B H}^{2}{ }^{4} H e} \propto \frac{(\text { nucl.eff } f)_{\mathcal{I}}}{(\text { nucl.eff. })_{B H}},
$$

i.e., it is proportional to the ratio of the nuclear effects on the $\mathrm{BH}$ and DVCS interference to the nuclear effects on the $\mathrm{BH}$ cross section. If the nuclear dynamics modifies $\mathcal{I}$ and the $T_{B H}^{2}$ in a different way, the effect can be big even if the parton structure of the bound proton does not change appreciably. Our analysis suggests that this 


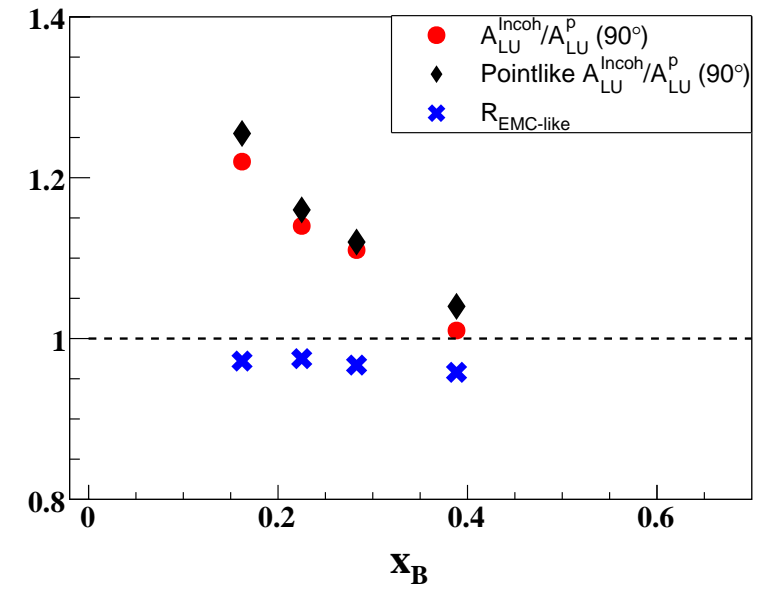

FIG. 4: (color online) The ratio $A_{L U}^{I n c o h} / A_{L U}^{p}$, Eq. (8) (red dots), compared to the result obtained with pointlike protons (black diamonds) and to the EMC-like ratio Eq. (9) (blue crosses).

is the case. This is seen in the other curves presented in Fig. 4. One of them, labelled "pointlike", is obtained considering in the ratio pointlike protons. Basically, the big effect is still there. Besides, in the same figure we show an "EMC-like" quantity, i.e., a ratio of a nuclear parton observable, the imaginary part of the $\mathrm{CFF}$, to the same observable for the free proton:

$$
R_{E M C-l i k e}=\frac{1}{\mathcal{N}} \frac{\int_{e x p} d E d \vec{p} P^{4} H e(\vec{p}, E) \Im m \mathcal{H}\left(\xi^{\prime}, \Delta^{2}\right)}{\Im m \mathcal{H}\left(\xi, \Delta^{2}\right)}
$$

where the factor $\mathcal{N}=\int_{\text {exp }} d E d \vec{p} P^{4} H e(\vec{p}, E)$ accounts for the fact that only a part of the spectral function is selected in a given experimental bin. One should notice that this ratio would be one if nuclear effects in the parton structure were negligible. As seen in Fig. 4 . this ratio is close to one and it resembles the EMC ratio, for ${ }^{4} \mathrm{He}$, at low $x_{B}$ [35. Since in our analysis the inner structure of the bound proton is entirely contained in the CFF and this produces a mild modification, the big effect found for the ratio (8), shown in Fig. 4, has little to do with a modification of the parton content. Rather, the effect is due to the different dependence on the 4-momentum components, affected by nuclear effects, of the interference and $\mathrm{BH}$ terms for the bound proton, or to other subtle effects.

Our thorough impulse approximation analysis, based on state-of-the-art models for the proton and nuclear structure, using a conventional description in terms of nucleon degrees of freedom, reproduces well the data on incoherent DVCS off ${ }^{4} \mathrm{He}$. This is true especially at high values of $Q^{2}$; the disagreement at low $Q^{2}$ points to possible FSI effects, to be investigated, or to other quark and gluon effects. While a benchmark calculation in the kine- matics of the next generation of precise measurements will require an improved treatment of both the nucleonic and the nuclear parts, such as a realistic evaluation of the spectral function and a test of further GPDs models, with an attempt to estimate the theoretical uncertainty of our predictions, the straightforward approach proposed here can be used as a workable framework for the planning of future measurements. Possible exotic quark and gluon effects in nuclei, not clearly seen within the present experimental accuracy, will be exposed by comparing forthcoming data with our conventional results.

We warmly thank R. Dupré and M. Hattawy for many helpful discussions and technical information and explanations on the EG6 experiment, and E. Voutier for insightful comments on the manuscript. This work was supported in part by the STRONG-2020 project of the European Union's Horizon 2020 research and innovation programme under grant agreement No 824093, and by the project "Deeply Virtual Compton Scattering off ${ }^{4} \mathrm{He}$ ", in the programme FRB of the University of Perugia.

[1] J. J. Aubert et al. [European Muon Collaboration], Phys. Lett. 123B 275 (1983).

[2] O. Hen, D. W. Higinbotham, G. A. Miller, E. Piasetzky and L. B. Weinstein, Int. J. Mod. Phys. E 221330017 (2013).

[3] R. Dupré and S. Scopetta, Eur. Phys. J. A 52 no.6, 159 (2016).

[4] I. C. Cloët et al., J. Phys. G 46, no. 9, 093001 (2019).

[5] D. Müller, D. Robaschik, B. Geyer, F.-M. Dittes and J. Hořejši, Fortsch. Phys. 42, 101 (1994); X. D. Ji, Phys. Rev. Lett. 78, 610 (1997); A. V. Radyushkin, Phys. Lett. B 380, 417 (1996).

[6] M. Diehl, Phys. Rept. 388, 41 (2003); A. V. Belitsky and A. V. Radyushkin, Phys. Rept. 418, 1 (2005).

[7] E. R. Berger, F. Cano, M. Diehl and B. Pire, Phys. Rev. Lett. 87142302 (2001).

[8] M. V. Polyakov and P. Schweitzer, Int. J. Mod. Phys. A 33, no. 26, 1830025 (2018) .

[9] M. Burkardt, Phys. Rev. D 62, 071503 (2000) Erratum: [Phys. Rev. D 66, 119903 (2002)] .

[10] A. Airapetian et al. [HERMES Collaboration], Phys. Rev. C 81, 035202 (2010).

[11] H. Egiyan, F.-X. Girod, K. Hafidi, S. Liuti, E. Voutier et al. Jefferson Lab Experiment E-08-024 (2008).

[12] M. Hattawy et al. [CLAS Collaboration], Phys. Rev. Lett. 119, no. 20, 202004 (2017).

[13] M. Hattawy et al. [CLAS Collaboration], Phys. Rev. Lett. 123, no. 3, 032502 (2019).

[14] W. R. Armstrong et al., arXiv:1708.00888 [nucl-ex].

[15] V. Guzey and M. Strikman, Phys. Rev. C 68, 015204 (2003); V. Guzey, A. W. Thomas and K. Tsushima, Phys. Lett. B 673, 9 (2009).

[16] S. Liuti and S. K. Taneja, Phys. Rev. C 72, 032201 (2005); Phys. Rev. C 72, 034902 (2005).

[17] S. Fucini, S. Scopetta and M. Viviani, Phys. Rev. C 98, 
no. 1,015203 (2018).

[18] K. Slifer et al. [E94010 Collaboration], Phys. Rev. Lett. 101, 022303 (2008).

[19] A. V. Belitsky, D. Mueller and A. Kirchner, Nucl. Phys. B 629, 323 (2002).

[20] S. Fucini, S. Scopetta and M. Viviani, in preparation.

[21] A. Del Dotto, E. Pace, G. Salmè and S. Scopetta, Phys. Rev. C 95, no. 1, 014001 (2017).

[22] A. V. Belitsky and D. Mueller, Phys. Rev. D 82, 074010 (2010).

[23] H. Morita and T. Suzuki, Prog. Theor. Phys. 86, 671 (1991).

[24] V. D. Efros, W. Leidemann and G. Orlandini, Phys. Rev. C 58, 582 (1998).

[25] M. Viviani, A. Kievsky and A. Rinat, Phys. Rev. C 67, 034003 (2003).

[26] A. S. Rinat, M. F. Taragin and M. Viviani, Phys. Rev. C 72, 015211 (2005).
[27] A. Kievsky, S. Rosati, M. Viviani, L. E. Marcucci and L. Girlanda, J. Phys. G 35, 063101 (2008).

[28] R. B. Wiringa, V. G. J. Stoks and R. Schiavilla, Phys. Rev. C 51, 38 (1995).

[29] B. S. Pudliner, V. R. Pandharipande, J. Carlson and R. B. Wiringa, Phys. Rev. Lett. 74, 4396 (1995).

[30] C. Ciofi degli Atti and S. Simula, Phys. Rev. C 53, 1689 (1996).

[31] S. Scopetta, Phys. Rev. C 79, 025207 (2009); M. Rinaldi and S. Scopetta, Phys. Rev. C 87, no. 3, 035208 (2013).

[32] S. V. Goloskokov and P. Kroll, Eur. Phys. J. C 50, 829 (2007).

[33] F. X. Girod et al. [CLAS Collaboration], Phys. Rev. Lett. 100, 162002 (2008).

[34] A. Kirchner and D. Mueller, Eur. Phys. J. C 32, 347 (2003).

[35] J. Seely et al., Phys. Rev. Lett. 103, 202301 (2009). 